\title{
Existence of anti-periodic solutions with symmetry for some high-order ordinary differential equations
}

Hai $\mathrm{Pu}^{1,2^{*}}$ and Jinyun Yang ${ }^{3}$

"Correspondence:
haipu_cumt@163.com
'State Key Laboratory for
Geomechanics and Deep
Underground Engineering, China
University of Mining and
Technology, Xuzhou, Jiangsu
221008, China
2School of Mechanics and Civil
Engineering, China University of
Mining and Technology, Xuzhou,
Jiangsu 221116, China
Full list of author information is
available at the end of the article

"Correspondence:

haipu_cumt@163.com

Laboratory for

Underground Engineering, China

University of Mining and

221008, China

${ }^{2}$ School of Mechanics and Civil thineering China Univery of Jiangsu 221116, China

available at the end of the article

\begin{abstract}
The existence of anti-periodic solutions with symmetry for high-order Duffing equations and a high-order Duffing type $p$-Laplacian equation has been studied by using degree theory. The results obtained enrich some known works to some extent. MSC: $34 \mathrm{~B} 15 ; 34 \mathrm{C} 25$

Keywords: anti-periodic solution with symmetry; high-order ordinary differential equation; $p$-Laplacian operator; Leray-Schauder degree theory
\end{abstract}

\section{Introduction}

Anti-periodic problems arise naturally from the mathematical models of various physical processes (see [1,2]) and also appear in the study of partial differential equations and abstract differential equations (see [3-5]). For instance, electron beam focusing system in traveling-wave tube theories is an anti-periodic problem (see [6]).

In mechanics, the simplest model of oscillation equation is a single pendulum equation

$$
x^{\prime \prime}+\omega^{2} \sin x=e(t)(\equiv e(t+2 \pi))
$$

whose anti-periodic solutions satisfy

$$
x(t+\pi)=-x(t), \quad \forall t \in \mathbb{R} .
$$

During the past twenty years, anti-periodic problems have been studied extensively by numerous scholars. For example, for first-order ordinary differential equations, a Massera's type criterion was presented in [7] and the validity of the monotone iterative technique was shown in [8]. Moreover, for higher-order ordinary differential equations, the existence of anti-periodic solutions was considered in [9-12]. Recently, existence results were extended to anti-periodic boundary value problems for impulsive differential equations (see [13]), and anti-periodic wavelets were discussed in [14].

It is well known that higher-order $p$-Laplacian equations are derived from many fields such as fluid mechanics and nonlinear elastic mechanics. In the past few decades, many important results on higher-order $p$-Laplacian equations with certain boundary conditions have been obtained. We refer the readers to [15-19] and the references cited therein.

\section{Springer}

(c) 2012 Pu and Yang: licensee Springer. This is an Open Access article distributed under the terms of the Creative Commons Attribution License (http://creativecommons.org/licenses/by/2.0), which permits unrestricted use, distribution, and reproduction in any medium, provided the original work is properly cited. 
In [10], the authors considered the existence of anti-periodic solutions for the high-order Duffing equation as follows:

$$
x^{(n)}+\sum_{i=1}^{n-1} a_{i} x^{(i)}+g(t, x)=e(t) .
$$

Moreover, in [15] the authors discussed the existence of anti-periodic solutions for the following higher-order Liénard type $p$-Laplacian equation:

$$
\left(\phi_{p}\left(x^{(n)}\right)\right)^{(n)}+f(x) x^{\prime}+g(t, x)=e(t)
$$

However, to the best of our knowledge, there exist relatively few results on the existence of anti-periodic solutions with symmetry for (1.1) and (1.2). Thus, it is worthwhile to continue to investigate the existence of anti-periodic solutions with symmetry for (1.1) and (1.2).

Motivated by the works mentioned previously, in this paper, we study the existence of anti-periodic solutions with symmetry for high-order Duffing equations of the forms:

$$
\begin{aligned}
& x^{(2 m+1)}+\sum_{i=1}^{m} a_{i} x^{(2 i-1)}+g(t, x)=e(t), \\
& x^{(2 m+2)}+\sum_{i=1}^{m} a_{i} x^{(2 i)}+g(t, x)=e(t)
\end{aligned}
$$

and high-order Duffing type $p$-Laplacian equation of the form:

$$
\left(\phi_{p}\left(x^{(m+1)}\right)\right)^{(m+1)}+g(t, x)=e(t),
$$

where $p>1$ is a constant, $m \geq 1$ is an integer, $\phi_{p}(s)=|s|^{p-2} s ; a_{i} \in \mathbb{R}, g \in C\left(\mathbb{R}^{2}, \mathbb{R}\right), e \in$ $C(\mathbb{R}, \mathbb{R})$ with $g(t+\pi,-x) \equiv-g(t, x), e(t+\pi) \equiv-e(t)$. Obviously, the inverse operator of $\phi_{p}$ is $\phi_{q}$, where $q>1$ is a constant such that $\frac{1}{p}+\frac{1}{q}=1$.

Notice that, when $p=2$, the nonlinear operator $\left(\phi_{p}\left(x^{(m+1)}\right)\right)^{(m+1)}$ reduces to the linear operator $x^{(2 m+2)}$. On the other hand, $x(t)$ is also a $2 \pi$-periodic solution if $x(t)$ is a $\pi$-antiperiodic solution. Hence, from the arguments in this paper, we can also obtain the existence results on periodic solutions for the above equations.

The rest of this paper is organized as follows. Section 2 contains some necessary preliminaries. In Section 3 and Section 4, basing on the Leray-Schauder principle, we establish some existence theorems on anti-periodic solutions with symmetry of (1.3), (1.4) and (1.5). Our results are different from those of bibliographies listed in the previous texts.

\section{Preliminaries}

For the sake of convenience, we set

$$
C^{k, \pi}=\left\{x \in C^{k}(\mathbb{R}, \mathbb{R}): x(t+\pi) \equiv-x(t)\right\}, \quad k \in\{0,1, \ldots\}
$$

with the norm

$$
\|x\|_{C^{k}}=\max _{i \in\{0,1, \ldots, k\}}\left\{\left\|x^{(i)}\right\|_{0}\right\}
$$


where $\|x\|_{0}=\max _{t \in[0,2 \pi]}|x(t)|$, and

$$
\begin{aligned}
& C_{0}^{k, \pi}=\left\{x \in C^{k, \pi}: x(-t) \equiv x(t)\right\}, \\
& C_{1}^{k, \pi}=\left\{x \in C^{k, \pi}: x(-t) \equiv-x(t)\right\}
\end{aligned}
$$

with the norm $\|\cdot\|_{C^{k}}$.

Notice that, $x \in C_{0}^{0, \pi}$ may be written as Fourier series as follows:

$$
x(t)=\sum_{i=0}^{\infty} a_{2 i+1} \cos (2 i+1) t
$$

and $x \in C_{1}^{0, \pi}$ may be written as the following Fourier series:

$$
x(t)=\sum_{i=0}^{\infty} b_{2 i+1} \sin (2 i+1) t
$$

where $a_{2 i+1}, b_{2 i+1} \in \mathbb{R}$. We define the mapping $J_{0}: C_{0}^{0, \pi} \longrightarrow C_{1}^{1, \pi}$ by

$$
\left(J_{0} x\right)(t)=\int_{0}^{t} x(s) d s=\sum_{i=0}^{\infty} \frac{a_{2 i+1}}{2 i+1} \sin (2 i+1) t, \quad \forall t \in \mathbb{R}
$$

and the mapping $J_{1}: C_{1}^{0, \pi} \longrightarrow C_{0}^{1, \pi}$ by

$$
\begin{aligned}
\left(J_{1} x\right)(t) & =\int_{0}^{t} x(s) d s-\sum_{i=0}^{\infty} \frac{b_{2 i+1}}{2 i+1} \\
& =-\sum_{i=0}^{\infty} \frac{b_{2 i+1}}{2 i+1} \cos (2 i+1) t, \quad \forall t \in \mathbb{R} .
\end{aligned}
$$

It is easy to prove that the mappings $J_{0}, J_{1}$ are completely continuous by using the ArzelàAscoli theorem.

Next, we introduce a continuation theorem (see [20]) as follows.

Lemma 2.1 (Continuation theorem) Let $\Omega$ be open bounded in a linear normal space $X$. Suppose that $f$ is a completely continuous field on $\bar{\Omega}$. Moreover, assume that the LeraySchauder degree

$$
\operatorname{deg}(f, \Omega, p) \neq 0, \quad \text { for } p \in X \backslash f(\partial \Omega) \text {. }
$$

Then the equation $f(x)=p$ has at least one solution in $\Omega$.

\section{Anti-periodic solutions with symmetry of (1.3) and (1.4)}

In this section, some existence results on anti-periodic solutions with symmetry of (1.3) and (1.4) will be given. 
$\left(\mathrm{H}_{1}\right)$ the functions $g(t, x)$ and $e(t)$ are odd in $t$, i.e.,

$$
g(-t, \cdot)=-g(t, \cdot), \quad e(-t)=-e(t), \quad \forall t \in \mathbb{R}
$$

$\left(\mathrm{H}_{2}\right)$ there exist non-negative functions $\alpha_{1}, \beta_{1} \in C\left(\mathbb{R}, \mathbb{R}^{+}\right)$such that

$$
|g(t, x)| \leq \alpha_{1}(t)|x|+\beta_{1}(t), \quad \forall t, x \in \mathbb{R}
$$

$\left(\mathrm{H}_{3}\right) \sum_{i=1}^{m}\left|a_{i}\right|+\left\|\alpha_{1}\right\|_{0}-1<0$

Then (1.3) has at least one even anti-periodic solution $x(t)$, i.e., $x(t)$ satisfies

$$
x(t+\pi)=-x(t), \quad x(-t)=x(t), \quad \forall t \in \mathbb{R} .
$$

Proof For making use of the Leray-Schauder degree theory to prove the existence of even anti-periodic solutions for (1.3), we consider the following homotopic equation of (1.3):

$$
x^{(2 m+1)}=-\lambda \sum_{i=1}^{m} a_{i} x^{(2 i-1)}-\lambda g(t, x)+\lambda e(t), \quad \lambda \in[0,1] .
$$

Define the operator $D_{01}: C_{0}^{2 m+1, \pi} \longrightarrow C_{1}^{0, \pi}$ by

$$
\left(D_{01} x\right)(t)=x^{(2 m+1)}(t), \quad \forall t \in \mathbb{R} .
$$

Obviously, the operator $D_{01}$ is invertible. Let $N_{01}: C_{0}^{2 m-1, \pi} \longrightarrow C^{0}$ be the Nemytskii operator

$$
\left(N_{01} x\right)(t)=-\sum_{i=1}^{m} a_{i} x^{(2 i-1)}(t)-g(t, x(t))+e(t), \quad \forall t \in \mathbb{R} .
$$

By hypothesis $\left(\mathrm{H}_{1}\right)$, it is easy to see that

$$
\left(N_{01} x\right)(t+\pi) \equiv-\left(N_{01} x\right)(t), \quad\left(N_{01} x\right)(-t) \equiv-\left(N_{01} x\right)(t), \quad \forall x \in C_{0}^{2 m-1, \pi}
$$

Thus, the operator $N_{01}$ sends $C_{0}^{2 m-1, \pi}$ into $C_{1}^{0, \pi}$. Hence, the problem of even anti-periodic solutions for (3.1) is equivalent to the operator equation

$$
D_{01} x=\lambda N_{01} x, \quad x \in C_{0}^{2 m+1, \pi} .
$$

From hypotheses $\left(\mathrm{H}_{2}\right),\left(\mathrm{H}_{3}\right)$ and (5) in [10], for the possible even anti-periodic solution $x(t)$ of (3.1), there exists a prior bounds in $C_{0}^{2 m+1, \pi}$, i.e., $x(t)$ satisfies

$$
\|x\|_{C^{2 m+1}} \leq T_{1}
$$

where $T_{1}$ is a positive constant independent of $\lambda$. So, our problem is reduced to construct one completely continuous operator $F_{\lambda}$, which sends $C_{0}^{2 m+1, \pi}$ into $C_{0}^{2 m+1, \pi}$, such that the 
fixed points of operator $F_{1}$ in some open bounded set are the even anti-periodic solutions of (1.3).

With this in mind, let us define the set as follows:

$$
\Omega_{01}=\left\{x \in C_{0}^{2 m+1, \pi}:\|x\|_{C^{2 m+1}}<T_{1}+1\right\} .
$$

Obviously, the set $\Omega_{01}$ is a open bounded set in $C_{0}^{2 m+1, \pi}$ and zero element $\theta \in \Omega_{01}$. Define the completely continuous operator $F_{\lambda}: \overline{\Omega_{01}} \longrightarrow C_{0}^{2 m+1, \pi}$ by

$$
F_{\lambda} x=\underbrace{J_{1} J_{0} \cdots J_{0} J_{1}}_{2 m+1} \lambda N_{01} x=\lambda D_{01}^{-1} N_{01} x, \quad \lambda \in[0,1] .
$$

Let us define the completely continuous field $h_{\lambda}(x): \overline{\Omega_{01}} \times[0,1] \longrightarrow C_{0}^{2 m+1, \pi}$ by

$$
h_{\lambda}(x)=x-F_{\lambda} x .
$$

By (3.2), we get that zero element $\theta \notin h_{\lambda}(\partial \Omega)$ for all $\lambda \in[0,1]$. So, the following LeraySchauder degrees are well defined and

$$
\begin{aligned}
\operatorname{deg}\left(i d-F_{1}, \Omega, \theta\right) & =\operatorname{deg}\left(h_{1}, \Omega, \theta\right) \\
& =\operatorname{deg}\left(h_{0}, \Omega, \theta\right)=\operatorname{deg}(i d, \Omega, \theta)=1 \neq 0 .
\end{aligned}
$$

Consequently, the operator $F_{1}$ has at least one fixed point in $\Omega_{01}$ by using Lemma 2.1. Namely, (1.3) has at least one even anti-periodic solution. The proof is complete.

Theorem 3.2 Assume that

$\left(\mathrm{H}_{4}\right)$ the function $g(t, x)$ is even in $t, x$ and $e(t)$ is even in $t$, i.e.,

$$
g(-t,-x)=g(t, x), \quad e(-t)=e(t), \quad \forall t \in \mathbb{R}
$$

and the assumptions $\left(\mathrm{H}_{2}\right),\left(\mathrm{H}_{3}\right)$ are true.

Then (1.3) has at least one odd anti-periodic solution $x(t)$, i.e., $x(t)$ satisfies

$$
x(t+\pi)=-x(t), \quad x(-t)=-x(t), \quad \forall t \in \mathbb{R} .
$$

Proof We consider the homotopic equation (3.1) of (1.3). Define the operator $D_{11}$ : $C_{1}^{2 m+1, \pi} \longrightarrow C_{0}^{0, \pi}$ by

$$
\left(D_{11} x\right)(t)=x^{(2 m+1)}(t), \quad \forall t \in \mathbb{R} .
$$

Let $N_{11}: C_{1}^{2 m-1, \pi} \longrightarrow C^{0, \pi}$ be the Nemytskii operator

$$
\left(N_{11} x\right)(t)=-\sum_{i=1}^{m} a_{i} x^{(2 i-1)}(t)-g(t, x(t))+e(t), \quad \forall t \in \mathbb{R} .
$$


By hypothesis $\left(\mathrm{H}_{4}\right)$, it is easy to see that

$$
\left(N_{11} x\right)(-t) \equiv\left(N_{11} x\right)(t), \quad \forall x \in C_{1}^{2 m-1, \pi}
$$

Thus, the operator $N_{11}$ sends $C_{1}^{2 m-1, \pi}$ into $C_{0}^{0, \pi}$. Hence, the problem of odd anti-periodic solutions for (3.1) is equivalent to the operator equation

$$
D_{11} x=\lambda N_{11} x, \quad x \in C_{1}^{2 m+1, \pi} .
$$

Our problem is reduced to construct one completely continuous operator $G_{\lambda}$, which sends $C_{1}^{2 m+1, \pi}$ into $C_{1}^{2 m+1, \pi}$, such that the fixed points of operator $G_{1}$ in some open bounded set are the odd anti-periodic solutions of (1.3). With this in mind, let us define the following set:

$$
\Omega_{11}=\left\{x \in C_{1}^{2 m+1, \pi}:\|x\|_{C^{2 m+1}}<T_{1}+1\right\} .
$$

Define the completely continuous operator $G_{\lambda}: \overline{\Omega_{11}} \longrightarrow C_{1}^{2 m+1, \pi}$ by

$$
G_{\lambda} x=\underbrace{J_{0} J_{1} \cdots J_{1} J_{0}}_{2 m+1} \lambda N_{11} x=\lambda D_{11}^{-1} N_{11} x, \quad \lambda \in[0,1] .
$$

The remainder of the proof work is quite similar to the proof of Theorem 3.1, so we omit the details. The proof is complete.

\section{Theorem 3.3 Assume that}

$\left(\mathrm{H}_{5}\right)$ the functions $g(t, x)$ and $e(t)$ are even in $t$, i.e.,

$$
g(-t, \cdot)=g(t, \cdot), \quad e(-t)=e(t), \quad \forall t \in \mathbb{R}
$$

and the assumptions $\left(\mathrm{H}_{2}\right),\left(\mathrm{H}_{3}\right)$ are true.

Then (1.4) has at least one even anti-periodic solution.

Proof We consider the homotopic equation of (1.4) as follows:

$$
x^{(2 m+2)}=-\lambda \sum_{i=1}^{m} a_{i} x^{(2 i)}-\lambda g(t, x)+\lambda e(t), \quad \lambda \in[0,1] .
$$

Define the operator $D_{02}: C_{0}^{2 m+2, \pi} \longrightarrow C_{0}^{0, \pi}$ by

$$
\left(D_{02} x\right)(t)=x^{(2 m+2)}(t), \quad \forall t \in \mathbb{R} .
$$

Let $N_{02}: C_{0}^{2 m, \pi} \longrightarrow C^{0, \pi}$ be the Nemytskii operator

$$
\left(N_{02} x\right)(t)=-\sum_{i=1}^{m} a_{i} x^{(2 i)}(t)-g(t, x(t))+e(t), \quad \forall t \in \mathbb{R}
$$


By hypothesis $\left(\mathrm{H}_{5}\right)$, it is easy to see that

$$
\left(N_{02} x\right)(-t) \equiv\left(N_{02} x\right)(t), \quad \forall x \in C_{0}^{2 m, \pi}
$$

Thus, the operator $N_{02}$ sends $C_{0}^{2 m, \pi}$ into $C_{0}^{0, \pi}$. Hence, the problem of even anti-periodic solutions for (3.3) is equivalent to the operator equation

$$
D_{02} x=\lambda N_{02} x, \quad x \in C_{0}^{2 m+2, \pi} .
$$

Our problem is reduced to construct one completely continuous operator $L_{\lambda}$, which sends $C_{0}^{2 m+2, \pi}$ into $C_{0}^{2 m+2, \pi}$, such that the fixed points of operator $L_{1}$ in some open bounded set are the even anti-periodic solutions of (1.4). With this in mind, let us define the following set:

$$
\Omega_{02}=\left\{x \in C_{0}^{2 m+2, \pi}:\|x\|_{C^{2 m+2}}<T_{2}+1\right\}
$$

where $T_{2}$ is a positive constant independent of $\lambda$. Define the completely continuous operator $L_{\lambda}: \overline{\Omega_{02}} \longrightarrow C_{0}^{2 m+2, \pi}$ by

$$
L_{\lambda} x=\underbrace{J_{1} J_{0} \cdots J_{1} J_{0}}_{2 m+2} \lambda N_{02} x=\lambda D_{02}^{-1} N_{02} x, \quad \lambda \in[0,1] .
$$

The remainder of the proof work is quite similar to the proof of Theorem 3.1, so we omit the details. The proof is complete.

\section{Theorem 3.4 Assume that}

$\left(\mathrm{H}_{6}\right)$ the function $g(t, x)$ is odd in $t, x$ and $e(t)$ is odd in $t$, i.e.,

$$
g(-t,-x)=-g(t, x), \quad e(-t)=-e(t), \quad \forall t \in \mathbb{R}
$$

and the assumptions $\left(\mathrm{H}_{2}\right),\left(\mathrm{H}_{3}\right)$ are true.

Then (1.4) has at least one odd anti-periodic solution.

Proof We consider the homotopic equation (3.3) of (1.4). Define the operator $D_{12}$ : $C_{1}^{2 m+2, \pi} \longrightarrow C_{1}^{0, \pi}$ by

$$
\left(D_{12} x\right)(t)=x^{(2 m+2)}(t), \quad \forall t \in \mathbb{R} .
$$

Let $N_{12}: C_{1}^{2 m, \pi} \longrightarrow C^{0, \pi}$ be the Nemytskii operator

$$
\left(N_{12} x\right)(t)=-\sum_{i=1}^{m} a_{i} x^{(2 i)}(t)-g(t, x(t))+e(t), \quad \forall t \in \mathbb{R}
$$

By hypothesis $\left(\mathrm{H}_{6}\right)$, it is easy to see that

$$
\left(N_{12} x\right)(-t) \equiv-\left(N_{12} x\right)(t), \quad \forall x \in C_{1}^{2 m, \pi}
$$


Thus, the operator $N_{12}$ sends $C_{1}^{2 m, \pi}$ into $C_{1}^{0, \pi}$. Hence, the problem of odd anti-periodic solutions for (3.3) is equivalent to the operator equation

$$
D_{12} x=\lambda N_{12} x, \quad x \in C_{1}^{2 m+2, \pi} .
$$

Our problem is reduced to construct one completely continuous operator $P_{\lambda}$ which sends $C_{1}^{2 m+2, \pi}$ into $C_{1}^{2 m+2, \pi}$, such that the fixed points of operator $P_{1}$ in some open bounded set are the odd anti-periodic solutions of (1.4). With this in mind, let us define the set as follows:

$$
\Omega_{12}=\left\{x \in C_{1}^{2 m+2, \pi}:\|x\|_{C^{2 m+2}}<T_{2}+1\right\} .
$$

Define the completely continuous operator $P_{\lambda}: \overline{\Omega_{12}} \longrightarrow C_{1}^{2 m+2, \pi}$ by

$$
P_{\lambda} x=\underbrace{J_{0} J_{1} \cdots J_{0} J_{1}}_{2 m+2} \lambda N_{12} x=\lambda D_{12}^{-1} N_{12} x, \quad \lambda \in[0,1] .
$$

The remainder of the proof work is quite similar to the proof of Theorem 3.1, so we omit the details. The proof is complete.

When $g(t, x)=g(x)$, we can remove the assumption $\left(\mathrm{H}_{2}\right)$ in Theorem 3.1, Theorem 3.2 and obtain the following results.

Theorem 3.5 Assume that

$\left(\mathrm{H}_{7}\right) \sum_{i=1}^{m}\left|a_{i}\right|-1<0$ and the assumption $\left(\mathrm{H}_{1}\right)$ is true.

Then (1.3) $(g(t, x)=g(x))$ has at least one even anti-periodic solution.

Theorem 3.6 Suppose that the assumptions $\left(\mathrm{H}_{4}\right),\left(\mathrm{H}_{7}\right)$ are true. Then $(1.3)(g(t, x)=g(x))$ has at least one odd anti-periodic solution.

Basing on the proof of Theorem 2 in [10], for the possible anti-periodic solution $x(t)$ of (3.1) $(g(t, x)=g(x))$, the hypothesis $\left(\mathrm{H}_{7}\right)$ yields that there exists a prior bounds in $C^{2 m+1, \pi}$, i.e., $x(t)$ satisfies

$$
\|x\|_{C^{2 m+1}} \leq T_{3}
$$

where $T_{3}$ is a positive constant independent of $\lambda$. The remainder of the proof work of Theorem 3.5 and Theorem 3.6 is quite similar to the proof of Theorem 3.1 and Theorem 3.2, so we omit the details.

\section{Anti-periodic solutions with symmetry of (1.5)}

In this section, we will give some existence results on anti-periodic solutions with symmetry of (1.5).

Theorem 4.1 Assume that 
$\left(\mathrm{H}_{8}\right)$ there exist non-negative functions $\alpha_{2}, \beta_{2} \in C\left(\mathbb{R}, \mathbb{R}^{+}\right)$such that

$$
|g(t, x)| \leq \alpha_{2}(t)|x|^{p-1}+\beta_{2}(t), \quad \forall t, x \in \mathbb{R}
$$

$\left(\mathrm{H}_{9}\right)\left\|\alpha_{2}\right\|_{0} \lambda_{1}^{-(m+1)}-1<0$ and the assumption $\left(\mathrm{H}_{5}\right)$ is true.

Then (1.5) has at least one even anti-periodic solution.

Proof We consider the following homotopic equation of (1.5):

$$
\left(\phi_{p}\left(x^{(m+1)}\right)\right)^{(m+1)}=-\lambda g(t, x)+\lambda e(t), \quad \lambda \in[0,1] .
$$

Define the operator $D_{03}: D\left(D_{03}\right) \subset C_{0}^{0, \pi} \longrightarrow L^{1}([0,2 \pi], \mathbb{R})$ by

$$
\left(D_{03} x\right)(t)=\left(\phi_{p}\left(x^{(m+1)}(t)\right)\right)^{(m+1)}, \quad \forall t \in \mathbb{R}
$$

where

$$
D\left(D_{03}\right)=\left\{x \in C_{0}^{2 m+1, \pi}:\left(\phi_{p}\left(x^{(m+1)}(t)\right)\right)^{(m)}\right.
$$

is absolutely continuous on $\mathbb{R}\}$.

Let $N_{03}: C_{0}^{0, \pi} \longrightarrow L^{1}([0,2 \pi], \mathbb{R})$ be the Nemytskii operator

$$
\left(N_{03} x\right)(t)=-g(t, x(t))+e(t), \quad \forall t \in \mathbb{R}
$$

Obviously, the operator $D_{03}$ is invertible and the problem of even anti-periodic solutions for (4.1) is equivalent to the operator equation

$$
D_{03} x=\lambda N_{03} x, \quad x \in D\left(D_{03}\right) .
$$

From hypotheses $\left(\mathrm{H}_{8}\right),\left(\mathrm{H}_{9}\right)$ and (3.8) in [15], for the possible even anti-periodic solution $x(t)$ of (4.1), there exists a prior bounds in $C_{0}^{0, \pi}$, i.e., $x(t)$ satisfies

$$
\|x\|_{C^{0}} \leq T_{4}
$$

where $T_{4}$ is a positive constant independent of $\lambda$. So, our problem is reduced to construct one completely continuous operator $Q_{\lambda}$, which sends $C_{0}^{0, \pi}$ into $C_{0}^{0, \pi}$, such that the fixed points of operator $Q_{1}$ in some open bounded set are the even anti-periodic solutions of (1.5).

With this in mind, let us define the set as follows:

$$
\Omega_{03}=\left\{x \in C_{0}^{0, \pi}:\|x\|_{C^{0}}<T_{4}+1\right\}
$$

By hypothesis $\left(\mathrm{H}_{5}\right)$, it is easy to see that

$$
\left(N_{03} x\right)(-t) \equiv\left(N_{03} x\right)(t), \quad \forall x \in C_{0}^{0, \pi} .
$$


Hence, the operator $N_{03}$ sends $C_{0}^{0, \pi}$ into $C_{0}^{0, \pi}$. Define the completely continuous operator $Q_{\lambda}: \overline{\Omega_{03}} \longrightarrow C_{0}^{0, \pi}$ by

$$
\begin{aligned}
Q_{\lambda} x & =\underbrace{J_{1} J_{0} \cdots J_{0} J_{1}}_{m+1} \phi_{q} \underbrace{J_{0} J_{1} \cdots J_{1} J_{0}}_{m+1} \lambda N_{03} x \\
& =\phi_{q}(\lambda) D_{03}^{-1} N_{03} x, \quad \lambda \in[0,1](\text { if } m=2 n, n=1,2, \ldots)
\end{aligned}
$$

or

$$
\begin{aligned}
Q_{\lambda} x & =\underbrace{J_{1} J_{0} \cdots J_{1} J_{0}}_{m+1} \phi_{q} \underbrace{J_{1}^{J_{0} \cdots J_{1} J_{0}} \lambda N_{03} x}_{m+1} \\
& =\phi_{q}(\lambda) D_{03}^{-1} N_{03} x, \quad \lambda \in[0,1](\text { if } m=2 n-1, n=1,2, \ldots) .
\end{aligned}
$$

The remainder of the proof work is quite similar to the proof of Theorem 3.1, so we omit the details. The proof is complete.

Theorem 4.2 Suppose that the assumptions $\left(\mathrm{H}_{6}\right),\left(\mathrm{H}_{8}\right),\left(\mathrm{H}_{9}\right)$ are true. Then $(1.5)$ has at least one odd anti-periodic solution.

Proof We consider the homotopic equation (4.1) of (1.5). Define the operator $D_{13}$ : $D\left(D_{13}\right) \subset C_{1}^{0, \pi} \longrightarrow L^{1}([0,2 \pi], \mathbb{R})$ by

$$
\left(D_{13} x\right)(t)=\left(\phi_{p}\left(x^{(m+1)}(t)\right)\right)^{(m+1)}, \quad \forall t \in \mathbb{R},
$$

where

$$
\begin{aligned}
D\left(D_{13}\right)= & \left\{x \in C_{1}^{2 m+1, \pi}:\left(\phi_{p}\left(x^{(m+1)}(t)\right)\right)^{(m)}\right. \\
& \text { is absolutely continuous on } \mathbb{R}\} .
\end{aligned}
$$

Let $N_{13}: C_{1}^{0, \pi} \longrightarrow L^{1}([0,2 \pi], \mathbb{R})$ be the Nemytskii operator

$$
\left(N_{13} x\right)(t)=-g(t, x(t))+e(t), \quad \forall t \in \mathbb{R}
$$

Thus, the problem of odd anti-periodic solutions for (4.1) is equivalent to the operator equation

$$
D_{13} x=\lambda N_{13} x, \quad x \in D\left(D_{13}\right) .
$$

Our problem is reduced to construct one completely continuous operator $W_{\lambda}$, which sends $C_{1}^{0, \pi}$ into $C_{1}^{0, \pi}$, such that the fixed points of operator $W_{1}$ in some open bounded set are the odd anti-periodic solutions of (1.5). With this in mind, let us define the following set:

$$
\Omega_{13}=\left\{x \in C_{1}^{0, \pi}:\|x\|_{C^{0}}<T_{4}+1\right\}
$$


By hypothesis $\left(\mathrm{H}_{6}\right)$, it is easy to see that

$$
\left(N_{13} x\right)(-t) \equiv-\left(N_{13} x\right)(t), \quad \forall x \in C_{1}^{0, \pi}
$$

Hence, the operator $N_{13}$ sends $C_{1}^{0, \pi}$ into $C_{1}^{0, \pi}$. Define the completely continuous operator $W_{\lambda}: \overline{\Omega_{13}} \longrightarrow C_{1}^{0, \pi}$ by

$$
\begin{aligned}
W_{\lambda} x & =\underbrace{J_{0} J_{1} \cdots J_{1} J_{0}}_{m+1} \phi_{q} \underbrace{J_{1} J_{0} \cdots J_{0} J_{1}}_{m+1} \lambda N_{13} x \\
& =\phi_{q}(\lambda) D_{13}^{-1} N_{13} x, \quad \lambda \in[0,1](\text { if } m=2 n, n=1,2, \ldots)
\end{aligned}
$$

or

$$
\begin{aligned}
W_{\lambda} x & =\underbrace{J_{0} J_{1} \cdots J_{0} J_{1}}_{m+1} \phi_{q} \underbrace{J_{0} J_{1} \cdots J_{0} J_{1}}_{m+1} \lambda N_{13} x \\
& =\phi_{q}(\lambda) D_{13}^{-1} N_{13} x, \quad \lambda \in[0,1](\text { if } m=2 n-1, n=1,2, \ldots) .
\end{aligned}
$$

The remainder of the proof work is quite similar to the proof of Theorem 3.1, so we omit the details. The proof is complete.

Theorem 4.3 Assume that $g(t, x)$ has the decomposition

$$
g(t, x)=u(t, x)+v(t, x)
$$

such that

$\left(\mathrm{H}_{10}\right)$ there exist non-negative constants $\gamma, r$ with $r>p$, such that

$$
(-1)^{m+1} x u(t, x) \geq \gamma|x|^{r}, \quad \forall t, x \in \mathbb{R}
$$

$\left(\mathrm{H}_{11}\right)$ there are non-negative functions $\alpha_{3}, \beta_{3} \in C\left(\mathbb{R}, \mathbb{R}^{+}\right)$such that

$$
|v(t, x)| \leq \alpha_{3}(t)|x|^{r-1}+\beta_{3}(t), \quad \forall t, x \in \mathbb{R}
$$

$\left(\mathrm{H}_{12}\right)\left\|\alpha_{3}\right\|_{0}-\gamma \leq 0$ and the assumption $\left(\mathrm{H}_{5}\right)$ is true.

Then (1.5) has at least one even anti-periodic solution.

Theorem 4.4 Suppose that the assumptions $\left(\mathrm{H}_{6}\right),\left(\mathrm{H}_{10}\right),\left(\mathrm{H}_{11}\right),\left(\mathrm{H}_{12}\right)$ are true. Then (1.5) has at least one odd anti-periodic solution.

Basing on the proof of Theorem 3.2 in [15], for the possible anti-periodic solution $x(t)$ of (4.1), the hypotheses $\left(\mathrm{H}_{10}\right),\left(\mathrm{H}_{11}\right),\left(\mathrm{H}_{12}\right)$ yield that there exists a prior bounds in $C^{2 m+1, \pi}$, i.e., $x(t)$ satisfies

$$
\|x\|_{C^{0}} \leq T_{5}
$$


where $T_{5}$ is a positive constant independent of $\lambda$. The remainder of the proof work of Theorem 4.3 and Theorem 4.4 is quite similar to the proof of Theorem 4.1 and Theorem 4.2, so we omit the details.

Remark Assumptions $\left(\mathrm{H}_{10}\right),\left(\mathrm{H}_{11}\right),\left(\mathrm{H}_{12}\right)$ guarantee that the degree with respect to $x$ of $g(t, x)$ is allowed to be greater than $p-1$, which is different from the hypothesis $\left(\mathrm{H}_{8}\right)$ of Theorem 4.1 and Theorem 4.2.

\section{Competing interests}

The authors declare that they have no competing interests.

\section{Authors' contributions}

HP carried out the theoretical analysis. JY drafted the manuscript. All authors read and approved the final manuscript.

\section{Author details}

'State Key Laboratory for Geomechanics and Deep Underground Engineering, China University of Mining and Technology, Xuzhou, Jiangsu 221008, China. ${ }^{2}$ School of Mechanics and Civil Engineering, China University of Mining and Technology, Xuzhou, Jiangsu 221116, China. ${ }^{3}$ School of Mathematics and Physical Sciences, Xuzhou Institute of Technology, Xuzhou, Jiangsu 221008, China.

\section{Acknowledgements}

This work was supported by the National Natural Science Foundations of China (50904065) and the Program for New Century Excellent Talents in University (NCET-09-0728). As well, this work was sponsored by the Qing Lan Project and the Fundamental Research Funds for the Central Universities (China University of Mining and Technology).

Received: 25 May 2012 Accepted: 24 September 2012 Published: 9 October 2012

\section{References}

1. Ahn, C, Rim, C: Boundary flows in general coset theories. J. Phys. A 32(13), 2509-2525 (1999)

2. Kleinert, H, Chervyakov, A: Functional determinants from Wronski Green function. J. Math. Phys. 40(11), 6044-6051 (1999)

3. Aizicovici, S, McKibben, M, Reich, S: Anti-periodic solutions to nonmonotone evolution equations with discontinuous nonlinearities. Nonlinear Anal. 43(2), 233-251 (2001)

4. Nakao, M: Existence of an anti-periodic solution for the quasilinear wave equation with viscosity. J. Math. Anal. Appl. 204(3), 754-764 (1996)

5. Souplet, P: Optimal uniqueness condition for the antiperiodic solutions of some nonlinear parabolic equations. Nonlinear Anal. 32(2), 279-286 (1998)

6. Lu, Z: Travelling Tube. Shanghai Sci. Technol., Shanghai (1962)

7. Chen, Y: On Massera's theorem for anti-periodic solution. Adv. Math. Sci. Appl. 9(1), 125-128 (1999)

8. Yin, Y: Monotone iterative technique and quasilinearization for some anti-periodic problems. Nonlinear World 3(2), 253-266 (1996)

9. Aftabizadeh, AR, Pavel, NH, Huang, Y: Anti-periodic oscillations of some second-order differential equations and optimal control problems. J. Comput. Appl. Math. 52(1-3), 3-21 (1994). (Oscillations in nonlinear systems: Applications and numerical aspects)

10. Chen, T, Liu, W, Zhang, J: The existence of anti-periodic solutions for high order Duffing equation. J. Appl. Math. Comput. 27(1-2), 271-280 (2008)

11. Liu, B: Anti-periodic solutions for forced Rayleigh-type equations. Nonlinear Anal., Real World Appl. 10(5), 2850-2856 (2009)

12. Liu, W, Zhang, J, Chen, T: Anti-symmetric periodic solutions for the third order differential systems. Appl. Math. Lett. 22(5), 668-673 (2009)

13. Luo, Z, Shen, J, Nieto, JJ: Antiperiodic boundary value problem for first-order impulsive ordinary differential equation. Comput. Math. Appl. 49(2-3), 253-261 (2005)

14. Chen, H: Antiperiodic wavelets. J. Comput. Math. 14(1), 32-39 (1996)

15. Chen, T, Liu, W: Anti-periodic solutions for higher-order Liénard type differential equation with $p$-Laplacian operator Bull. Korean Math. Soc. 49(3), 455-463 (2012)

16. Li, X: Existence and uniqueness of periodic solutions for a kind of high-order $p$-Laplacian Duffing differential equation with sign-changing coefficient ahead of linear term. Nonlinear Anal. 71(7-8), 2764-2770 (2009)

17. Pang, H, Ge, W, Tian, M: Solvability of nonlocal boundary value problems for ordinary differential equation of higher order with a p-Laplacian. Comput. Math. Appl. 56(1), 127-142 (2008)

18. Su, H, Wang, B, Wei, Z, Zhang, X: Positive solutions of four-point boundary value problems for higher-order p-Laplacian operator. J. Math. Anal. Appl. 330(2), 836-851 (2007)

19. $\mathrm{Xu}, \mathrm{F}, \mathrm{Liu}, \mathrm{L}, \mathrm{Wu}, \mathrm{Y}$ : Multiple positive solutions of four-point nonlinear boundary value problems for a higher-order p-Laplacian operator with all derivatives. Nonlinear Anal. 71(9), 4309-4319 (2009)

20. Deimling, K: Nonlinear Functional Analysis. Springer, Berlin (1985) 
doi:10.1186/1687-2770-2012-108

Cite this article as: Pu and Yang: Existence of anti-periodic solutions with symmetry for some high-order ordinary differential equations. Boundary Value Problems 2012 2012:108.

Submit your manuscript to a SpringerOpen ${ }^{\circ}$ journal and benefit from:

- Convenient online submission

- Rigorous peer review

- Immediate publication on acceptance

- Open access: articles freely available online

- High visibility within the field

- Retaining the copyright to your article

Submit your next manuscript at $\gg$ springeropen.com 